\title{
Honamli Goats Breed in South of Turkey I- Serum Mineral Analysis ${ }^{\#}$
}

\author{
Ayşe Özge Demir ${ }^{1, a, *}$, Kıvanç Irak ${ }^{2, b}$, Handan Mert ${ }^{3, c}$, Nihat Mert ${ }^{3, d}$, Nesrullah Ayşinn ${ }^{4, e}$, Inci Sogutlu ${ }^{5, f}$ \\ ${ }^{1}$ Department of Animal Science, Faculty of Agriculture, Van Yuzuncu Yil University, 65080 Van, Turkey \\ ${ }^{2}$ Department of Biochemistry, Faculty of Veterinary Medicine, Siirt University, 56100 Siirt, Turkey \\ ${ }^{3}$ Department of Biochemistry, Faculty of Veterinary Medicine, Van Yuzuncu Yil University, 65080 Van, Turkey \\ ${ }^{4}$ Department of Vocational School of Health Services, Hakkari University,30000 Hakkari, Turkey \\ ${ }^{5}$ Republic of Turkey Ministry of Agriculture and Foresty, 06800 Ankara, Turkey \\ *Corresponding author
}

\section{A R T I C L E I N F O}

${ }^{\#}$ This study was presented as poster at the XXVII. National Biochemistry Congress, Antalya, Turkey, 03-06

November 2015, ss:39

Research Article

Received : 22/04/2020 Accepted : 09/09/2020

\section{Keywords:}

Blood minerals

Honamli Goat

TIBC

Transferrin

UIBC

\section{A B S T R A C T}

The objectivity of this study was to investigate some blood minerals and parameters in Honamli goats reared under semi-intensive conditions. 90 goats (2-4 ages) in different three herds kept under similar manage mental conditions were chosen as research materials in Teke Border of Antalya Province. Serum Ca 6.786 $\pm 0.206 \mathrm{mg} / \mathrm{dL}, \mathrm{P} 4.094 \pm 0.173 \mathrm{mg} / \mathrm{dL}, \mathrm{Cl} 111.105 \pm 0.582 \mathrm{mmol} / \mathrm{L}, \mathrm{K}$ $4.519 \pm 0.066 \mathrm{mmol} / \mathrm{L}, \mathrm{Mg} 2.161 \pm 0.05 \mathrm{mg} / \mathrm{dL}, \mathrm{Na} 148.047 \pm 0.508 \mathrm{mmol} / \mathrm{L}, \mathrm{Fe} 110.706 \pm 2.510$ $\mu \mathrm{g} / \mathrm{dL}$, UIBC $155.025 \pm 4.333 \mu \mathrm{g} / \mathrm{dL}$ and $\mathrm{Tp} 7.055 \pm 0.120 \mathrm{~g} / \mathrm{dL}$ were determined by Roche Diagnostics, Cobas 8000 modular analyser series, immunoassay. After then, total iron- binding capacity (TIBC), transferrin (Tf), transferrin saturation (TSAT) and Ca++ levels were calculated from each other using different biochemical formulas. Ratios between the some minerals were calculated and presented in the text. Also, very high significant differences $(\mathrm{P}<0.0001)$ were found between herds for $\mathrm{P}, \mathrm{Cl}$, TIBC and Tf. However, no significant differences were found for $\mathrm{Ca}, \mathrm{Na}$, TSAT, TP and Ca results. Coefficients of variation (CV) were within $2 \%$ and $35 \%$ for all outcome parameters. In Pearson correlation analysis, negative and different two significant values $(\mathrm{P}<0.01$ and $\mathrm{P}<0.001$ ) were calculated between $\mathrm{Fe}$ and unsaturated iron binding capacity (UIBC) for all analysed groups. Controversially, positive and very high significant correlations $(\mathrm{P}<0.0001)$ were detected between $\mathrm{Cl}$ and $\mathrm{Na}$. The results obtained from this study could serve as reference values for Honamli goats breeding in Mediterranean Region of Turkey. \begin{tabular}{ll}
\hline & (iD) http://orcid.org/0000-0001-7203-4734 \\
aodemir@yyu.edu.tr & (i) http://orcid.org/0000-0001-9827-7996
\end{tabular} e@nesrullahaysin@hu.edu.tr
(iD) http://orcid.org/0000-0001-7087-8810

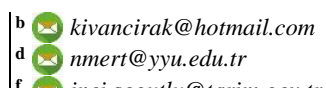

nmert@yyu.edu.tr

inci.sogutlu@tarim.gov.tr http://orcid.org/0000-0001-9765-0330 D http://orcid.org/0000-0001-7185-3316 (iD) http://orcid.org/0000-0002-9957-4738

(c) (1) () T) This work is licensed under Creative Commons Attribution 4.0 International License

\section{Introduction}

Quite a large number of known minerals that necessary for breeding of sheep (Ovis aries) and goat (Capra hircus) are responsible for ensuring the proper orderly functioning of organisms (Ogunleke et al., 2014). When the level of minerals in the blood falls below normal, all of the organism's resistance, health, functioning and production mechanisms are gradually affected (Anonymous, 2018a).

It is generally accepted that the formation of mineral deficiency depends on 3 main factors. Firstly, the most common cause of deficiencies is inadequate quality of traditional grazing meadows (Kawas et al., 2010; Xin et al., 2011). Secondary causes of mineral deficiencies are known as deficiencies caused by absorption problems in the digestive system and the adverse effects of some other minerals. $\mathrm{Mg}$ is in efficiently absorbed from the rumen.
High Mg deficiency causes Grass tetany (Lactation tetany) in sheeps and goats. As an example of tertiary cause, it can be shown that the amount of $\mathrm{K}$ in the diet and the amount of excess $\mathrm{Ca}$ affect the $\mathrm{Mg}$ absorption. The other an important point related with body cover of sheep and goat, similar to some other animals' cover, the appearance and analysis values of wool, hair and mohair are indicative of the mineral level of these organism. Also this situation contains reflects especially both food and nutrition quality (Patkowska-Sokola et al., 2009). The fleece-eating, associated with mineral imbalances and deficiencies, is quite common an important health problem leading financial losses in small ruminants. This clinical disease is particularly located at very low levels of blood S and Mo in small ruminants. If the organism has these inadequacies, 
it may need to be identified at some other important mineral levels such as $\mathrm{Ca}, \mathrm{P}, \mathrm{Fe}, \mathrm{Mn}, \mathrm{Zn}, \mathrm{Cu}, \mathrm{Co}$ and $\mathrm{Se}$ (Patkowska-Sokola et al., 2009). Because in consequence this inadequacy in minerals instinctively creates an act of eating wooll in small ruminants.

As an another example of interaction between minerals is Fe. The Fe, a necessary trace element, involved in a large number of biological processes (Dlouhy and Outten, 2013; Yee and Tolman, 2015) is the effective in transition processes in metabolism in all living organisms. From other side, Fe level in blood is important because of prevents uptake of other some minerals that are in very small quantities, such as $\mathrm{Zn}$. Because minerals interact with each other and with other food items. In the meantime, excessive intake of a mineral can cause the lack of another food item. For these reasons, it is necessary to be sure that ruminant animals are healthy, to know their various blood mineral levels and to be use cation when nutritional supplement is necessary. In the case of such a requirement, only a healthy interpretation can be made when the reference intervals for blood mineral levels are known.

This study was carried out in Antalya (36 $46^{\circ} 12.728^{\prime \prime}$ $\mathrm{N}$ and $31^{\circ} 26^{\prime} 28.615^{\prime \prime} \mathrm{E}$ ) located in the Teke region and at elevation 61 meters above sea level, where Honamli goats are grown extensively. This geographical region includes both Antalya and Burdur, Isparta, Mugla, Denizli. Honamli goats are heavily cultivated by the nomads in this region. The Honamli nomads spend winter months in the Mediterranean region, especially in the provinces of Antalya's districts: Serik, Manavgat, Alanya and spend summer months on the Toros Plateau and south of Konya as wandering. The Mediterranean climate that dominates this region is dependent on the Summer heats, the Sun rays, the drought and downward air movements. In addition, annual temperature average is $18{ }^{\circ} \mathrm{C}$, snow and frost are very rare in winter months. While amount of maximum rainfall occurs in winter months, amount of minimum level rainfall occurs in summer months. Actually, the plant cover in this area is the forest. But, Makis are formed by the destruction of this forests in low areas $(0-800 \mathrm{~m})$. Maki is a plant community that can tolerate summer drought and grows from short dwarf trees like Mersin, Laurel, Olive, Oleander, Carob.

This study was conducted in February month when, Honamli goats gain to the most benefit from the Maki plant coverings and had high amounts of milk yield. In this way, some mineral values of healthy Honamli goats raised under native husbandry practice in Antalya Teke Plateau calculated and presented. Thus, it is aimed to serve as a reference source for future work.

\section{Materials and Methods}

Randomly selected 30 Honamli goat, from among 2-4 years old, known be healthy and free of external, internal and blood parasites bred at the three different herds were main material of this study. Also, each herds had similar maintenance and feeding conditions.

Blood samples were collected from goats in order to see and associate with blood minerals values. For this purpose, standard method was done during the blood collection process, and blood samples were taken from the goats via jugular venipuncture using a $5 \mathrm{~mL}$ syringe. These samples were then transferred in tubes without anticoagulants for serum removed. Samples guarded in the cold chain were brought to the laboratory to be centrifuged. In the laboratory, centrifugation was performed at standard room temperature for 10 minutes at $1500 \mathrm{~g}$. Obtained serums by this process were transferred to polypropylene micro centrifuge tubes for subsequent biochemical analyses and stored at $-20^{\circ} \mathrm{C}$ in the deep freeze.

Mineral values were determined at ppm level using Roche Diagnostics, Cobas 8000 modular analyser series (Bieglmayer et al., 2004) in the biochemistry laboratory at the University of Health Sciences, Education and Research Hospital in Van, Turkey. For this purpose, concentrations of the substances to be identified were made by subtracting the measurement curve after calibrating using the device's standard concentration values. Standard solutions were used for calibration procedures (Demir et al., 2011). After then, total iron-binding capacity (TIBC), transferrin (Tf), transferrin saturation (TSAT) and $\mathrm{Ca}++$ levels were calculated from each other using different biochemical formulas (Anonymous, 2018b; c) as reported by Kunish and Small (1970) and were presented in the below.

$$
\begin{aligned}
& \mathrm{TIBC}=\mathrm{Fe}+\mathrm{UIBC} \\
& \mathrm{Ca}++=(\mathrm{Ca} \times 6-\mathrm{TP} / 3) / \mathrm{TP}+6(\text { Raphael, } 1983) \\
& \mathrm{TSAT}=\mathrm{Fe} / \mathrm{TIBC} \times 100 \\
& \mathrm{Tf} \quad=\mathrm{Fe} \times 71.24 / \mathrm{TSAT}
\end{aligned}
$$

However, calculated results to be in accordance with the literature were presented as $\mathrm{mg} / \mathrm{dL}$ for $\mathrm{Ca}, \mathrm{P}, \mathrm{Mg}$; $\mu \mathrm{g} / \mathrm{dL}$ for $\mathrm{Fe}$; $\mathrm{mmol} / \mathrm{l}$ for $\mathrm{Cl}, \mathrm{K}$ and $\mathrm{Na}$. Data were analysed using variance procedure (SPSS Base 7.5 for Windows 1997). Significant differences at a probability of $(\mathrm{P}<0.05)$ were compared using Duncan's procedure of the same software. Then, the relationships between minerals were analyzed to put forward for revealing with Pearson Correlation Coefficient at the significant level of $\mathrm{P}<0.05$.

\section{Results}

Mean concentration \pm standard error $(\mathrm{x} \pm \mathrm{Sx})$, minimum-maximum (min-max), median and $\mathrm{CV}$ values (\%) of serum minerals and their derivatives were presented in Table 1. When the mineral values were examined, it was determined that there was no statistical significant $(\mathrm{P}>0.05)$ between the groups in terms of $\mathrm{Ca}, \mathrm{Na}, \mathrm{TSAT}, \mathrm{TP}$ and $\mathrm{Ca}++$ values, while three different level statistical significant between the groups were significant in $\mathrm{K}$ and Fe levels $(\mathrm{P}<0.05)$; $\mathrm{Mg}$ and UIBC level $(\mathrm{P}<0.01) ; \mathrm{P}, \mathrm{Cl}$, TIBC and Tf level $(\mathrm{P}<0.001)$. It was determined that the highest values of parameters except $P$ and $K$ were in the 1 st herd. At the statistically significant parameters, while the $\mathrm{Fe}(67.768 \pm 0.438 \mathrm{mg} / \mathrm{dL})$ value of total herd was close to the value of 1st herd, all of the other mineral's ( $\mathrm{P}, \mathrm{Cl}, \mathrm{K}$, $\mathrm{Mg}$, UIBC, TIBC, Tf) values were close to the values of 2nd herd. According to these results, most of the values of the total herd can be considered to represent with 2nd herd's values. Moreover, when the median values were examined, the 2nd herd had the closest values to the total herd for $\mathrm{P}$, $\mathrm{K}, \mathrm{Mg}, \mathrm{Fe}, \mathrm{UIBC}$, TIBC, TSAT and Tf. Furthermore, the $\mathrm{CV} \%$ (Coefficient of variation) values has been found undesirably high levels except $\mathrm{Cl}(4.884 \%)$ and $\mathrm{Na}$ (3.197 $\%$ ) in this study (Table 1). 
Table 1. Mineral levels and derivatives in serum samples

\begin{tabular}{|c|c|c|c|c|c|c|c|}
\hline & Herd & $\mathrm{N}$ & Mean \pm Std. E & Min-Max & Median & $\% \mathrm{CV}$ & $\mathrm{P}$ \\
\hline \multirow{4}{*}{$\begin{array}{c}\mathrm{Ca} \\
\mathrm{mg} / \mathrm{dl}\end{array}$} & 1 & 25 & $7.342 \pm 0.299^{\mathrm{a}}$ & $4.510-9.580$ & 7.260 & 20.335 & \multirow{4}{*}{$0.101^{\mathrm{NS}}$} \\
\hline & 2 & 29 & $6.295 \pm 0324^{\mathrm{a}}$ & $1.680-9.940$ & 6.410 & 27.673 & \\
\hline & 3 & 22 & $6.804 \pm 0.438^{\mathrm{a}}$ & $2.560-9.800$ & 6.970 & 30.199 & \\
\hline & Total & 76 & $6.786 \pm 0.206$ & $1.680-9.940$ & 6.830 & 26.460 & \\
\hline \multirow{4}{*}{$\begin{array}{c}\mathrm{P} \\
\mathrm{mg} / \mathrm{dl}\end{array}$} & 1 & 29 & $3.197 \pm 0.111^{\mathrm{c}}$ & $2.130-4.510$ & 3.190 & 18.649 & \multirow{4}{*}{$0.0001 * * *$} \\
\hline & 2 & 29 & $4.036 \pm 0.164^{\mathrm{b}}$ & $2.900-6.700$ & 3.930 & 21.814 & \\
\hline & 3 & 13 & $6.222 \pm 0.476^{\mathrm{a}}$ & $2.740-8.930$ & 5.680 & 27.593 & \\
\hline & Total & 71 & $4.0935 \pm 0.173$ & $2.1300-8.930$ & 3.660 & 35.677 & \\
\hline \multirow{4}{*}{$\begin{array}{c}\mathrm{Cl} \\
\mathrm{mmol} / 1\end{array}$} & 1 & 30 & $114.830 \pm 1.175^{\mathrm{a}}$ & $106.700-140.600$ & 114.050 & 5.604 & \multirow{4}{*}{$0.0001 * * *$} \\
\hline & 2 & 30 & $109.260 \pm 0.654^{\mathrm{b}}$ & $102.400-118.600$ & 108.750 & 3.278 & \\
\hline & 3 & 27 & $109.015 \pm 0.678^{b}$ & $96.000-116.300$ & 109.000 & 3.233 & \\
\hline & Total & 87 & $111.105 \pm 0.582$ & $96.000-140.600$ & 109.800 & 4.884 & \\
\hline \multirow{4}{*}{$\underset{\mathrm{mmol} / \mathrm{l}}{\mathrm{K}}$} & 1 & 30 & $4.269 \pm 0.075^{\mathrm{b}}$ & $3.420-5.180$ & 4.240 & 9.658 & \multirow{4}{*}{$0.0143 *$} \\
\hline & 2 & 30 & $4.583 \pm 0.148^{\mathrm{a}}$ & $3.250-6.350$ & 4.490 & 17.694 & \\
\hline & 3 & 27 & $4.729 \pm 0.090^{\mathrm{a}}$ & $4.060-5.890$ & 4.670 & 9.910 & \\
\hline & Total & 87 & $4.519 \pm 0.066$ & $3.250-6.350$ & 4.460 & 13.679 & \\
\hline \multirow{4}{*}{$\underset{\mathrm{mg} / \mathrm{dl}}{\mathrm{Mg}}$} & 1 & 27 & $2.366 \pm 0.105^{\mathrm{a}}$ & $0.010-3.010$ & 2.400 & 22.965 & \multirow{4}{*}{$0.0034 * *$} \\
\hline & 2 & 27 & $2.169 \pm 0.073^{\mathrm{a}}$ & $1.280-2.910$ & 2.230 & 17.541 & \\
\hline & 3 & 22 & $1.900 \pm 0.095^{\mathrm{b}}$ & $1.060-2.650$ & 2.060 & 23.484 & \\
\hline & Total & 76 & $2.161 \pm 0.057$ & 0.010-3.010 & 2.255 & 22.828 & \\
\hline \multirow{4}{*}{$\begin{array}{c}\mathrm{Na} \\
\mathrm{mmol} / \mathrm{l}\end{array}$} & 1 & 30 & $148.903 \pm 1.173^{\mathrm{a}}$ & $142.200-176.000$ & 147.800 & 4.314 & \multirow{4}{*}{$0.2437^{\mathrm{NS}}$} \\
\hline & 2 & 30 & $146.907 \pm 0.590^{\mathrm{a}}$ & $137.400-155.700$ & 146.800 & 2.197 & \\
\hline & 3 & 29 & $148.363 \pm 0.721^{\mathrm{a}}$ & $132.000-154.100$ & 148.800 & 2.524 & \\
\hline & Total & 89 & $148.047 \pm 0.508$ & $132.000-176.000$ & 147.900 & 3.197 & \\
\hline \multirow{4}{*}{$\begin{array}{c}\mathrm{Fe} \\
\mu \mathrm{g} / \mathrm{dl}\end{array}$} & 1 & 29 & $112.786 \pm 3.762^{\mathrm{a}}$ & $67.600-144.100$ & 115.100 & 17.963 & \multirow{4}{*}{$0.0166^{*}$} \\
\hline & 2 & 29 & $115.479 \pm 3.932^{\mathrm{a}}$ & $77.800-175.000$ & 111.500 & 18.337 & \\
\hline & 3 & 14 & $96.507 \pm 4.996^{\mathrm{b}}$ & $64.600-130.600$ & 99.050 & 19.370 & \\
\hline & Total & 72 & $110.706 \pm 2.510$ & $64.600-175.000$ & 110.950 & 19.236 & \\
\hline \multirow{4}{*}{$\begin{array}{r}\mathrm{UIBC} \\
\mu \mathrm{g} / \mathrm{dl}\end{array}$} & 1 & 29 & $165.735 \pm 6.524^{\mathrm{a}}$ & $107.500-230.800$ & 165.400 & 21.200 & \multirow{4}{*}{$0.0031 * *$} \\
\hline & 2 & 30 & $160.037 \pm 6.984^{\mathrm{a}}$ & $61.300-240.500$ & 155.600 & 23.902 & \\
\hline & 3 & 18 & $129.417 \pm 7.454^{\mathrm{b}}$ & $67.100-175.400$ & 131.200 & 24.436 & \\
\hline & Total & 77 & $155.025 \pm 4.333$ & $61.300-250.500$ & 155.800 & 24.527 & \\
\hline \multirow{4}{*}{$\begin{array}{l}\text { TIBC } \\
\mu \mathrm{g} / \mathrm{dl}\end{array}$} & 1 & 28 & $278.796 \pm 5.857^{\mathrm{a}}$ & $211.700-344.700$ & 290.400 & 11.116 & \multirow{4}{*}{$0.0001 * * *$} \\
\hline & 2 & 29 & $276.648 \pm 6.572^{\mathrm{a}}$ & $217.100-361.400$ & 274.300 & 12.793 & \\
\hline & 3 & 14 & $224.186 \pm 7.613^{b}$ & $166.400-269.800$ & 227.450 & 12.706 & \\
\hline & Total & 71 & $267.151 \pm 4.570$ & $166.400-361.400$ & 269.800 & 14.415 & \\
\hline \multirow{4}{*}{$\begin{array}{c}\text { TSAT } \\
\%\end{array}$} & 1 & 28 & $40.911 \pm 1.543^{\mathrm{a}}$ & $22.930-54.040$ & 42.505 & 19.956 & \\
\hline & 2 & 29 & $42.253 \pm 1.670^{\mathrm{a}}$ & $24.840-74.060$ & 41.860 & 21.286 & \\
\hline & 3 & 14 & $43.599 \pm 2.540^{\mathrm{a}}$ & $28.050-59.680$ & 44.895 & 21.798 & $0.6332^{\mathrm{NS}}$ \\
\hline & Total & 71 & $41.989 \pm 1.034$ & $22.930-74.060$ & 41.960 & 20.742 & \\
\hline & 1 & 28 & $180.037 \pm 4.685^{\mathrm{a}}$ & $126.360-232.760$ & 189.320 & 13.771 & \\
\hline $\mathrm{Tf}$ & 2 & 29 & $178.319 \pm 5.258^{a}$ & $130.680-246.120$ & 176.440 & 15.878 & \\
\hline $\mathrm{g} / \mathrm{l}$ & 3 & 13 & $136.686 \pm 6.568^{b}$ & $90.120-172.840$ & 140.360 & 17.326 & $0.0001 * * *$ \\
\hline & Total & 70 & $171.274 \pm 3.666$ & $90.120-246.120$ & 172.840 & 17.908 & \\
\hline & 1 & 29 & $7.242 \pm 0.115^{\mathrm{a}}$ & $7.210-8.780$ & 7.180 & 8.526 & \\
\hline $\mathrm{TP}$ & 2 & 29 & $6.722 \pm 0.125^{\mathrm{a}}$ & $4.270-8.270$ & 6.700 & 9.978 & \\
\hline $\mathrm{g} / \mathrm{dl}$ & 3 & 26 & $7.218 \pm 0.298^{a}$ & $2.730-9.410$ & 7.625 & 21.033 & $0.0911^{\mathrm{NS}}$ \\
\hline & Total & 84 & $7.055 \pm 0.120$ & $2.730-9.410$ & 7.120 & 14.412 & \\
\hline & 1 & 25 & $3.135 \pm 0.119^{\mathrm{a}}$ & $1.992-4.014$ & 3.201 & 18.957 & \\
\hline & 2 & 27 & $2.878 \pm 0.129^{\mathrm{a}}$ & $1.624-4.253$ & 2.813 & 23.328 & $2160 \mathrm{NS}$ \\
\hline $\mathrm{Ca}^{\mathrm{TT}}$ & 3 & 22 & $2.838 \pm 0.199^{\mathrm{a}}$ & $0.924-4.437$ & 2.904 & 32.943 & $0.3169^{103}$ \\
\hline & Total & 74 & $2.953 \pm 0.086$ & $0.924-4.437$ & 2.925 & 25.012 & \\
\hline
\end{tabular}

$\mathrm{P}<0.001,{ }^{\mathrm{a}, \mathrm{b}, \mathrm{c}}$ Differences between the values involving different letters in the same column were found to be statistically significant at $\mathrm{P}<0.05$

The results of mineral ratios of this study were shown in Table 2. According to this, the proportional values of the 6 minerals ( $\mathrm{Ca}, \mathrm{P}, \mathrm{Mg}, \mathrm{Na}, \mathrm{K}, \mathrm{Fe})$ known to be effected at high levels of each other were presented as 4 groups $(\mathrm{Ca} / \mathrm{P}$, $\mathrm{Ca} / \mathrm{Mg}, \mathrm{Na} / \mathrm{K}, \mathrm{P} / \mathrm{Fe}$ ). Moreover, while $\mathrm{Ca} / \mathrm{P}, \mathrm{Ca} / \mathrm{Mg}, \mathrm{P} / \mathrm{Fe}$ ratios obtained by making necessary calculations were presented in $\mathrm{mg} / \mathrm{dL}, \mathrm{Na} / \mathrm{K}$ in $\mathrm{mmol} / \mathrm{L}$ in Table 2 . In the presented study, the most common mineral proportions in terms of biochemical interactions were calculated as $\mathrm{Ca} / \mathrm{P}$ 1.666, $\mathrm{Ca} / \mathrm{Mg} 3.233, \mathrm{Na} / \mathrm{K} 32.792$ and $\mathrm{P} / \mathrm{Fe} 42.51$.
Mineral ranking of 7 minerals analysed in the serum samples according to the obtained results were shown in Table 3. Ranking, on the converted value in $\mathrm{mg} / \mathrm{dL}$ was made by calculating. Also, it was seen that the mineral rating was the same for all herds. According to this, $\mathrm{Cl}$ was at the highest level, while Fe was at the lowest level at all herds. And rankings were as follows: $\mathrm{Cl}>\mathrm{Na}>\mathrm{K}>\mathrm{Ca}>\mathrm{P}>\mathrm{Mg}>\mathrm{Fe}$

In Table 4, the results of correlation of the data obtained from this study were presented. In this instance, 
correlations were analyzed for each herd and total. 78 main correlation groups were seen in the presented correlation table. Positive statistical significances at the highest level $(\mathrm{P}<0.001)$ were between $\mathrm{Ca}-\mathrm{Ca}++, \mathrm{P}-\mathrm{K}, \mathrm{Cl}-\mathrm{Na}, \mathrm{Fe}-\mathrm{TSAT}$, UIBC-TIBC, UIBC-Tf and TIBC-Tf. Also, when the table was examined, it was clear that there were negative correlations as well as positive correlations. According to this, negative statistical significances at the highest level
$(\mathrm{P}<0.001)$ were between UIBC-TSAT, TIBC-TSAT, TSAT-Tf. Besides, low level positive correlations $(\mathrm{P}<0.05)$ were between Ca-TP, Cl-TIBC, Cl-Tf, Na-Fe, Fe-TIBC and Fe-Tf. The other hand, different levels negative statistical significant in total herd were between $\mathrm{P}-\mathrm{Cl}, \mathrm{Fe}-$ UIBC $(\mathrm{P}<0.05)$; UIBC-TSAT $(\mathrm{P}<0.001)$; TBC-TSAT, TSAT-Tf $(\mathrm{P}<0.001)$.

Table 2. Mineral proportions in serum samples

\begin{tabular}{l|cccccccc}
\hline \multirow{2}{*}{ Herd } & \multicolumn{7}{c}{ Mineral proportion } \\
\cline { 2 - 9 } & $\mathrm{N}$ & $\mathrm{Ca} / \mathrm{P}$ & $\mathrm{N}$ & $\mathrm{Ca} / \mathrm{Mg}$ & $\mathrm{N}$ & $\mathrm{Na} / \mathrm{K}$ & $\mathrm{N}$ & $\mathrm{P} / \mathrm{Fe}$ \\
\hline & & $\mathrm{mg} / \mathrm{dl}$ & & $\mathrm{mg} / \mathrm{dl}$ & & $\mathrm{mmol} / \mathrm{l}$ & $\mathrm{mg} / \mathrm{dl}$ \\
1 & 25 & 2.319 & 25 & 3.144 & 30 & 34.880 & 29 & 28.292 \\
2 & 28 & 1.551 & 26 & 2.999 & 30 & 32.055 & 29 & 35.096 \\
3 & 11 & 1.077 & 23 & 3.600 & 27 & 31.538 & 11 & 64.144 \\
\hline Total & 64 & 1.666 & 74 & 3.233 & 87 & 32.792 & 69 & 42.511 \\
\hline
\end{tabular}

Table 3. Mineral ranking in serum samples

\begin{tabular}{|c|c|c|c|c|c|c|c|c|c|c|c|c|c|}
\hline \multirow{2}{*}{ Herd } & \multicolumn{13}{|c|}{ Mineral ranking (mg/dl) } \\
\hline & 1 & & 2 & & 3 & & 4 & & 5 & & 6 & & 7 \\
\hline 1 & $\mathrm{Cl}$ & $>$ & $\mathrm{Na}$ & $>$ & K & $>$ & $\mathrm{Ca}$ & $>$ & $\mathrm{P}$ & $>$ & $\mathrm{Mg}$ & $>$ & $\mathrm{Fe}$ \\
\hline 2 & $\mathrm{Cl}$ & $>$ & $\mathrm{Na}$ & $>$ & K & $>$ & $\mathrm{Ca}$ & $>$ & $\mathrm{P}$ & $>$ & $\mathrm{Mg}$ & $>$ & $\mathrm{Fe}$ \\
\hline 3 & $\mathrm{Cl}$ & $>$ & $\mathrm{Na}$ & $>$ & K & $>$ & $\mathrm{Ca}$ & $>$ & $\mathrm{P}$ & $>$ & $\mathrm{Mg}$ & $>$ & $\mathrm{Fe}$ \\
\hline Total & $\mathrm{Cl}$ & $>$ & $\mathrm{Na}$ & $>$ & $\mathrm{K}$ & $>$ & $\mathrm{Ca}$ & $>$ & $\mathrm{P}$ & $>$ & $\mathrm{Mg}$ & $>$ & $\mathrm{Fe}$ \\
\hline
\end{tabular}

\section{Discussion and Conclusion}

Some of the topics such as morphological characteristics (Alizadehasl and Unal, 2011; Elmaz et al., 2012a; Gok et al., 2015), descriptive characteristics (Gok et al., 2011; Elmaz et al., 2012), improvement studies (Gok et al., 2014a), genetic (Gok et al., 2011; Gok et al., 2014b) and fattening performance-carcass characteristics (Aktas et al., 2015) have been studied on Honamli goats by some researchers in the last 10 years. Despite that, the only study of the biochemical values of Honamli goats belonged to Devrim et al. (2015). These researchers studied on the values of alkaline phosphatase (ALP), alanine transaminase (ALT), aspartate transaminase (AST), creatine kinase $(\mathrm{CK})$, calcium, cholesterol, creatinine, iron, magnesium, phosphorus, total lipids, triglycerides and uric acid on Honamli and Native Hair goats during pubertal development. In this presented study, the results obtained and the varieties presented by them are extensively mentioned.

Calcium: In this study, the mean Ca value of the total herd was $6.786 \pm 0.438 \mathrm{mg} / \mathrm{dL}$. Moreover, the minimummaximum Ca values ranged from 1.680 to $9.940 \mathrm{mg} / \mathrm{dL}$, and no statistical significant $(\mathrm{P}>0.05)$ was between 3 Honamli herds.

Devrim et al. (2015) reported that the Ca values in 12 months old Honamli goats were $11.21 \pm 0.19 \mathrm{mg} / \mathrm{dL}$. It was also transferred that no statistical significant $(\mathrm{P}>0.05)$ found in Ca level in 4, 8 and 12 months old Honamli goats. Sovende et al. (2008), reported as Ca $5.39 \pm 0.21 \mathrm{mmol} / \mathrm{L}$ the result of blood minerals of Wad goats grazing in the natural pastures. In another study (Fujihara et al., 2006) conducted blood mineral concentration of grazing goats in Luzon Island of Philippines, it was reported that Ca level as $94.58 \pm 2.61 \mathrm{mg} / \mathrm{L}$ and $100.03 \pm 3.34 \mathrm{mg} / \mathrm{L}$ in the dry and wet season, respectively. But $\mathrm{Ca}$ result presented by Fujihara et al. (2006) were higher than critical level reported by McDowell (1985) in the same study as Ca 90 $\mathrm{mg} / \mathrm{L}$. While the level of $\mathrm{Ca}$ in this study was lower than study conducted by Devrim et al (2015), it was higher than Sovende et al (2008). On the other hand, Ca level in the total of 3 herds was found to be lower compared to the one reported by Fujihara et al. (2006), while result was higher than critical level reported by McDowell (1985). Also, no statistical significant $(\mathrm{P}>0.05)$ between 3 Honamli herds were found like as a similar result of Devrim et al. (2015).

Phosphor: In this presented study, the mean $\mathrm{P}$ value of the total herd was $4.094 \pm 0.173 \mathrm{mg} / \mathrm{dL}$. Moreover, the minimum-maximum $P$ values ranged from 2.130 to 8.930 $\mathrm{mg} / \mathrm{dL}$, and a fairly high level statistical significant $(\mathrm{P}<0.001)$ was between 3 Honamli herds.

Devrim et al. (2015) state that P levels in 12 months old Honamli goats were as $6.43 \pm 0.33 \mathrm{mg} / \mathrm{dL}$ in they study. In addition, a statistical significant $(\mathrm{P}<0.05)$ between 4,8 and 12 months old Honamli goats has been reported. Sovende et al. (2008) reported the result of $\mathrm{P}$ of Wad goats as $3.48 \pm 0.12 \mathrm{mmol} / \mathrm{L}$. On the other hand, Fujihara et al. (2006) examined blood mineral levels of goats in Luzon Island of Philippines and, they were reported that $\mathrm{P}$ levels were $65.14 \pm 3.38 \mathrm{mg} / \mathrm{L}$ and $63.59 \pm 3.37 \mathrm{mg} / \mathrm{L}$ in the dry and wet season, respectively. In relation to this mineral results obtained from the study, $\mathrm{P}$ levels were higher than reported critical level P $40 \mathrm{mg} / \mathrm{L}$ by McDowell (1985) in the same study. However, the $\mathrm{P}$ value of this presented study is very close to the values reported by Sovende et al. (2008) and reported as critical by McDowell (1985). However, defined $\mathrm{P}$ value was also lower than both the value declared by Devrim et al. (2015) and studied on Wad goats by Fujihara et al. (2006). Whereas, $P$ level in the total of 3 herds in this study was found to be lower compared to the ones reported by Fujihara et al. (2006). 
Table 4. Correlation levels of minerals and derivatives in serum samples

\begin{tabular}{|c|c|c|c|c|c|c|c|c|c|c|c|c|c|}
\hline & $\mathrm{H}$ & $\mathrm{N}$ & $\mathrm{P}$ & $\mathrm{N}$ & $\mathrm{Cl}$ & $\mathrm{N}$ & $\mathrm{K}$ & $\mathrm{N}$ & $\mathrm{Mg}$ & $\mathrm{N}$ & $\mathrm{Na}$ & $\mathrm{N}$ & $\mathrm{Fe}$ \\
\hline \multirow{4}{*}{$\mathrm{Ca}$} & 1 & 25 & $-0.218^{\mathrm{NS}}$ & 25 & $-0.273^{\mathrm{NS}}$ & 25 & $-0.197^{\mathrm{NS}}$ & 25 & $-0.243^{\mathrm{NS}}$ & 25 & $-0.465^{*}$ & 25 & $-0.391 *$ \\
\hline & 2 & 28 & $-0.132^{\mathrm{NS}}$ & 29 & $0.236^{\mathrm{NS}}$ & 29 & $0.211^{\mathrm{NS}}$ & 27 & $0.145^{\mathrm{NS}}$ & 29 & $0.102^{\mathrm{NS}}$ & 28 & $0.367^{\mathrm{NS}}$ \\
\hline & 3 & 10 & $0.240^{\mathrm{NS}}$ & 22 & $-0.043^{\mathrm{NS}}$ & 22 & $0.2629^{\mathrm{NS}}$ & 21 & $0.320^{\mathrm{NS}}$ & 22 & $0.106^{\mathrm{NS}}$ & 12 & $0.370^{\mathrm{NS}}$ \\
\hline & $\mathrm{T}$ & 63 & $-0.163^{\mathrm{NS}}$ & 76 & $0.066^{\mathrm{NS}}$ & 76 & $0.072^{\mathrm{NS}}$ & 73 & $0.076^{\mathrm{NS}}$ & 76 & $-0.080^{\mathrm{NS}}$ & 65 & $0.077^{\mathrm{NS}}$ \\
\hline \multirow{4}{*}{$\mathrm{P}$} & 1 & & & 29 & $-0.060^{\mathrm{NS}}$ & 29 & $0.360^{\mathrm{NS}}$ & 27 & $0.115^{\mathrm{NS}}$ & 29 & $0.129^{\mathrm{NS}}$ & 29 & $0.242^{\mathrm{NS}}$ \\
\hline & 2 & & & 29 & $-0.132^{\mathrm{NS}}$ & 29 & $0.281^{\mathrm{NS}}$ & 26 & $-0.001^{\mathrm{NS}}$ & 29 & $0.116^{\mathrm{NS}}$ & 29 & $0.088^{\mathrm{NS}}$ \\
\hline & 3 & & 1.000 & 13 & $0.147^{\mathrm{NS}}$ & 13 & $0.815^{* * *}$ & 9 & $0.280^{\mathrm{NS}}$ & 13 & $0.337^{\mathrm{NS}}$ & 10 & $0.306^{\mathrm{NS}}$ \\
\hline & $\mathrm{T}$ & & & 71 & $-0.0250 *$ & 71 & $0.425 * * *$ & 62 & $-0.203^{\mathrm{NS}}$ & 71 & $0.114^{\mathrm{NS}}$ & 68 & $-0.098^{\mathrm{NS}}$ \\
\hline \multirow{4}{*}{$\mathrm{Cl}$} & 1 & & & & & 30 & $0.523 * *$ & 27 & $-0.012^{\mathrm{NS}}$ & 30 & $0.895^{* * *} *$ & 29 & $0.196^{\mathrm{NS}}$ \\
\hline & 2 & & & & & 28 & $0.192^{\mathrm{NS}}$ & 28 & $0.290^{\mathrm{NS}}$ & 26 & $0.757 * * *$ & 28 & $0.379 *$ \\
\hline & 3 & & & & 1.000 & 27 & $0.322^{\mathrm{NS}}$ & 22 & $-0.043^{\mathrm{NS}}$ & 27 & $0.842 * * *$ & 14 & $0.049^{\mathrm{NS}}$ \\
\hline & $\mathrm{T}$ & & & & & 87 & $0.094^{\mathrm{NS}}$ & 76 & $0.196^{\mathrm{NS}}$ & 87 & $0.795^{* * *}$ & 72 & $0.226^{\mathrm{NS}}$ \\
\hline \multirow{4}{*}{$\mathrm{K}$} & 1 & & & & & & & 27 & $0.280^{\mathrm{NS}}$ & 30 & $0.560 * * *$ & 29 & $0.184^{\mathrm{NS}}$ \\
\hline & 2 & & & & & & & 27 & $-0.361^{\mathrm{NS}}$ & 30 & $-0.010^{\mathrm{NS}}$ & 29 & $0.249^{\mathrm{NS}}$ \\
\hline & 3 & & & & & & 1.000 & 22 & $-0.138^{\mathrm{NS}}$ & 27 & $0.269^{\mathrm{NS}}$ & 14 & $0.278^{\mathrm{NS}}$ \\
\hline & $\mathrm{T}$ & & & & & & & 76 & $-0.181^{\mathrm{NS}}$ & 87 & $0.190^{\mathrm{NS}}$ & 72 & $0.176^{\mathrm{NS}}$ \\
\hline & 1 & & & & & & & & & 27 & $0.177^{\mathrm{NS}}$ & 27 & $0.370^{\mathrm{NS}}$ \\
\hline & 2 & & & & & & & & & 27 & $0.340^{\mathrm{NS}}$ & 26 & $0.012^{\mathrm{NS}}$ \\
\hline $\mathrm{Mg}$ & 3 & & & & & & & & 1.000 & 22 & $0.058^{\mathrm{NS}}$ & 12 & $-0.494^{\mathrm{NS}}$ \\
\hline & $\mathrm{T}$ & & & & & & & & & 76 & $0.183^{\mathrm{NS}}$ & 65 & $0.193^{\mathrm{NS}}$ \\
\hline & 1 & & & & & & & & & & & 29 & $0.352^{\mathrm{NS}}$ \\
\hline & 2 & & & & & & & & & & & 29 & $0.457 *$ \\
\hline $\mathrm{Na}$ & 3 & & & & & & & & & & 1.000 & 14 & $0.233^{\mathrm{NS}}$ \\
\hline & $\mathrm{T}$ & & & & & & & & & & & 72 & $0.301 *$ \\
\hline & 1 & & & & & & & & & & & & \\
\hline $\mathrm{Fe}$ & 2 & & & & & & & & & & & & 1.000 \\
\hline Fe & $\begin{array}{l}3 \\
\mathrm{~T}\end{array}$ & & & & & & & & & & & & 1.000 \\
\hline & $\mathrm{H}$ & $\mathrm{N}$ & UIBC & $\mathrm{N}$ & TIBC & $\mathrm{N}$ & TSAT & $\mathrm{N}$ & Tf & $\mathrm{N}$ & TP & $\mathrm{N}$ & $\mathrm{Ca}++$ \\
\hline & 1 & 25 & $0.380^{\mathrm{NS}}$ & 24 & $0.200^{\mathrm{NS}}$ & 24 & $-0.456^{*}$ & 24 & $0.200^{\mathrm{NS}}$ & 25 & $0.613^{* * *}$ & 25 & $0.976^{* * * *}$ \\
\hline & 2 & 29 & $-0.144^{\mathrm{NS}}$ & 28 & $0.077^{\mathrm{NS}}$ & 28 & $0.266^{\mathrm{NS}}$ & 28 & $0.077^{\mathrm{NS}}$ & 28 & $0.404^{*}$ & 27 & $0.985^{* * * *}$ \\
\hline $\mathrm{Ca}$ & 3 & 14 & $-0.110^{\mathrm{NS}}$ & 12 & $0.240^{\mathrm{NS}}$ & 12 & $0.163^{\mathrm{NS}}$ & 11 & $0.255^{\mathrm{NS}}$ & 22 & $-0.104^{\mathrm{NS}}$ & 22 & $0.977 * * *$ \\
\hline & $\mathrm{T}$ & 68 & $0.067^{\mathrm{NS}}$ & 64 & $0.118^{\mathrm{NS}}$ & 64 & $-0.000^{\mathrm{NS}}$ & 63 & $0.141^{\mathrm{NS}}$ & 75 & $0.267^{*}$ & 74 & $0.975^{* * *} *$ \\
\hline & 1 & 29 & $0.080^{\mathrm{NS}}$ & 28 & $0.249^{\mathrm{NS}}$ & 28 & $0.059^{\mathrm{NS}}$ & 28 & $0.250^{\mathrm{NS}}$ & 29 & $0.176^{\mathrm{NS}}$ & 25 & $-0.300^{\mathrm{NS}}$ \\
\hline & 2 & 29 & $0.343^{\mathrm{NS}}$ & 29 & $0.426^{*}$ & 29 & $-0.191^{\mathrm{NS}}$ & 29 & $0.426^{*}$ & 28 & $-0.419^{*}$ & 26 & $0.227^{\mathrm{NS}}$ \\
\hline $\mathrm{P}$ & 3 & 13 & $-0.415^{\mathrm{NS}}$ & 10 & $-0.098^{\mathrm{NS}}$ & 10 & $0.416^{\mathrm{NS}}$ & 10 & $-0.098^{\mathrm{NS}}$ & 13 & $-0.530^{\mathrm{NS}}$ & 10 & $0.212^{\mathrm{NS}}$ \\
\hline & $\mathrm{T}$ & 71 & $-0.171^{\mathrm{NS}}$ & 67 & $-0.113^{\mathrm{NS}}$ & 67 & $-0.036^{\mathrm{NS}}$ & 67 & $-0.113^{\mathrm{NS}}$ & 70 & $-0.313^{* *}$ & 61 & $-0.121^{\mathrm{NS}}$ \\
\hline & 1 & 29 & $0.010^{\mathrm{NS}}$ & 28 & $0.138^{\mathrm{NS}}$ & 28 & $0.069^{\mathrm{NS}}$ & 28 & $0.138^{\mathrm{NS}}$ & 29 & $0.200^{\mathrm{NS}}$ & 25 & $-0.338^{\mathrm{NS}}$ \\
\hline & 2 & 28 & $-0.042^{\mathrm{NS}}$ & 28 & $0.176^{\mathrm{NS}}$ & 28 & $0.241^{\mathrm{NS}}$ & 28 & $0.176^{\mathrm{NS}}$ & 27 & $0.069^{\mathrm{NS}}$ & 26 & $0.299^{\mathrm{NS}}$ \\
\hline $\mathrm{Cl}$ & 3 & 18 & $0.056^{\mathrm{NS}}$ & 14 & $0.361^{\mathrm{NS}}$ & 14 & $-0.130^{\mathrm{NS}}$ & 13 & $0.365^{\mathrm{NS}}$ & 26 & $-0.165^{\mathrm{NS}}$ & 22 & $-0.066^{\mathrm{NS}}$ \\
\hline & $\mathrm{T}$ & 77 & $0.105^{\mathrm{NS}}$ & 71 & $0.251^{*}$ & 71 & $0.023^{\mathrm{NS}}$ & 70 & $0.248^{*}$ & 84 & $0.074^{\mathrm{NS}}$ & 74 & $0.032^{\mathrm{NS}}$ \\
\hline & 1 & 29 & $0.178^{\mathrm{NS}}$ & 28 & $0.327^{\mathrm{NS}}$ & 28 & $-0.041^{\mathrm{NS}}$ & 28 & $0.327^{\mathrm{NS}}$ & 29 & $0.211^{\mathrm{NS}}$ & 25 & $-0.266^{\mathrm{NS}}$ \\
\hline & 2 & 30 & $0.284^{\mathrm{NS}}$ & 29 & $0.440^{*}$ & 29 & $-0.024^{\mathrm{NS}}$ & 29 & $0.440^{*}$ & 29 & $0.143^{\mathrm{NS}}$ & 27 & $0.205^{\mathrm{NS}}$ \\
\hline $\mathrm{K}$ & 3 & 18 & $-0.352^{\mathrm{NS}}$ & 14 & $-0.250^{\mathrm{NS}}$ & 14 & $0.415^{\mathrm{NS}}$ & 13 & $-0.252^{\mathrm{NS}}$ & 26 & $-0.484 *$ & 22 & $0.229^{\mathrm{NS}}$ \\
\hline & $\mathrm{T}$ & 77 & $0.031^{\mathrm{NS}}$ & 71 & $0.187^{\mathrm{NS}}$ & 71 & $0.057^{\mathrm{NS}}$ & 70 & $0.213^{\mathrm{NS}}$ & 84 & $-0.113^{\mathrm{NS}}$ & 74 & $0.050^{\mathrm{NS}}$ \\
\hline & 1 & 27 & $-0.160^{\mathrm{NS}}$ & 26 & $0.065^{\mathrm{NS}}$ & 26 & $0.293^{\mathrm{NS}}$ & 26 & $0.065^{\mathrm{NS}}$ & 27 & $-0.134^{\mathrm{NS}}$ & 25 & $-0.234^{\mathrm{NS}}$ \\
\hline & 2 & 27 & $-0.095^{\mathrm{NS}}$ & 26 & $-0.335^{\mathrm{NS}}$ & 26 & $0.006^{\mathrm{NS}}$ & 26 & $-0.034^{\mathrm{NS}}$ & 27 & $0.093^{\mathrm{NS}}$ & 26 & $0.136^{\mathrm{NS}}$ \\
\hline $\mathrm{Mg}$ & 3 & 14 & $0.123^{\mathrm{NS}}$ & 12 & $-0.274^{\mathrm{NS}}$ & 12 & $-0.282^{\mathrm{NS}}$ & 11 & $-0.277^{\mathrm{NS}}$ & 22 & $-0.066^{\mathrm{NS}}$ & 21 & $0.314^{\mathrm{NS}}$ \\
\hline & $\mathrm{T}$ & 68 & $0.078^{\mathrm{NS}}$ & 64 & $0.208^{\mathrm{NS}}$ & 64 & $0.011^{\mathrm{NS}}$ & 63 & $0.208^{\mathrm{NS}}$ & 76 & $-0.114^{\mathrm{NS}}$ & 72 & $0.100^{\mathrm{NS}}$ \\
\hline & 1 & 29 & $-0.094^{\mathrm{NS}}$ & 28 & $0.122^{\mathrm{NS}}$ & 28 & $0.226^{\mathrm{NS}}$ & 28 & $0.122^{\mathrm{NS}}$ & 29 & $0.116^{\mathrm{NS}}$ & 25 & $-0.516 * *$ \\
\hline & 2 & 30 & $-0.159^{\mathrm{NS}}$ & 29 & $0.088^{\mathrm{NS}}$ & 29 & $0.361^{\mathrm{NS}}$ & 29 & $0.088^{\mathrm{NS}}$ & 29 & $0.017^{\mathrm{NS}}$ & 27 & $0.280^{\mathrm{NS}}$ \\
\hline $\mathrm{Na}$ & 3 & 18 & $0.232^{\mathrm{NS}}$ & 14 & $0.579 *$ & 14 & $-0.119^{\mathrm{NS}}$ & 13 & $0.585^{*}$ & 26 & $-0.034^{\mathrm{NS}}$ & 22 & $0.042^{\mathrm{NS}}$ \\
\hline & $\mathrm{T}$ & 77 & $-0.056^{\mathrm{NS}}$ & 71 & $0.137^{\mathrm{NS}}$ & 71 & $0.167^{\mathrm{NS}}$ & 70 & $0.144^{\mathrm{NS}}$ & 84 & $0.066^{\mathrm{NS}}$ & 74 & $-0.089^{\mathrm{NS}}$ \\
\hline & 1 & 29 & $-0.503 * *$ & 28 & $0.084^{\mathrm{NS}}$ & 28 & $0.819 * * *$ & 28 & $0.084^{\mathrm{NS}}$ & 29 & $0.007^{\mathrm{NS}}$ & 25 & $-0.432 *$ \\
\hline & 2 & 29 & $-0.413^{*}$ & 29 & $0.150^{\mathrm{NS}}$ & 29 & $0.801 * * *$ & 29 & $0.150^{\mathrm{NS}}$ & 28 & $0.082^{\mathrm{NS}}$ & 26 & $0.479 *$ \\
\hline $\mathrm{Fe}$ & 3 & 14 & $-0.458 *$ & 14 & $0.147^{\mathrm{NS}}$ & 14 & $0.781 * * *$ & 13 & $0.150^{\mathrm{NS}}$ & 14 & $0.1113^{\mathrm{NS}}$ & 12 & $0.358^{\mathrm{NS}}$ \\
\hline & $\mathrm{T}$ & 72 & $-0.277 *$ & 71 & $0.280^{*}$ & 71 & $0.723 * * *$ & 70 & $0.274^{*}$ & 71 & $-0.097^{\mathrm{NS}}$ & 63 & $0.154^{\mathrm{NS}}$ \\
\hline & 1 & & & 28 & $0.818^{* * * *}$ & 28 & $-0.905^{* * *}$ & 28 & $0.818^{* * *} *$ & 29 & $0.316^{\mathrm{NS}}$ & 25 & $0.357^{\mathrm{NS}}$ \\
\hline IIP & 2 & & $10 \Omega 0$ & 29 & $0.838 * * *$ & 29 & $-0.866^{* * *}$ & 29 & $0.838 * * *$ & 29 & $-0.121^{\mathrm{NS}}$ & 27 & $-0.080^{\mathrm{NS}}$ \\
\hline UIBC & 3 & & 1.000 & 14 & $0.812 * * *$ & 14 & $-9.03 * * *$ & 13 & $0.812 * * *$ & 18 & $0.367^{\mathrm{NS}}$ & 14 & $-0.185^{\mathrm{NS}}$ \\
\hline & $\mathrm{T}$ & & & 71 & $0.845^{* * *}$ & 71 & $-0.850 * * *$ & 70 & $0.843^{* * *}$ & 76 & $0.139^{\mathrm{NS}}$ & 66 & $0.109^{\mathrm{NS}}$ \\
\hline & 1 & & & & & 28 & $-0.499 * *$ & 28 & $1.000^{* * *}$ & 28 & $0.388^{*}$ & 24 & $0.135^{\mathrm{NS}}$ \\
\hline & 2 & & & & & 29 & $-0.461 *$ & 29 & $1.000^{* * *}$ & 28 & $-0.013^{\mathrm{NS}}$ & 26 & $0.200^{\mathrm{NS}}$ \\
\hline TIBC & 3 & & & & 1.000 & 14 & $-0.492^{\mathrm{NS}}$ & 13 & $1.000^{* * * *}$ & 14 & $0.543^{*}$ & 12 & $0.141^{\mathrm{NS}}$ \\
\hline & $\mathrm{T}$ & & & & & 71 & $-0.447 * * *$ & 70 & $1.000^{* * * *}$ & 70 & $-0.033^{\mathrm{NS}}$ & 62 & $0.208^{\mathrm{NS}}$ \\
\hline & 1 & & & & & & & 28 & $-0.499 * *$ & 28 & $-0.208^{\mathrm{NS}}$ & 24 & $-0.458^{N S}$ \\
\hline & 2 & & & & & & & 29 & $-0.461 *$ & 28 & $0.082^{\mathrm{NS}}$ & 26 & $0.283^{\mathrm{NS}}$ \\
\hline TSAT & 3 & & & & & & 1.000 & 13 & $-0.491^{\mathrm{NS}}$ & 14 & $-0.245^{\mathrm{NS}}$ & 12 & $0.220^{\mathrm{NS}}$ \\
\hline & $\mathrm{T}$ & & & & & & & 70 & $-0.446 * * *$ & 70 & $-0.073^{\mathrm{NS}}$ & 62 & $0.014^{\mathrm{NS}}$ \\
\hline & 1 & & & & & & & & & 28 & $0.388^{*}$ & 24 & $0.135^{\mathrm{NS}}$ \\
\hline & 2 & & & & & & & & 1000 & 28 & $-0.013^{\mathrm{NS}}$ & 26 & $0.200^{\mathrm{NS}}$ \\
\hline Tf & 3 & & & & & & & & 1.000 & 13 & $0.712^{* * *}$ & 11 & $0.146^{\mathrm{NS}}$ \\
\hline & $\mathrm{T}$ & & & & & & & & & 69 & $0.018^{\mathrm{NS}}$ & 61 & $0.219^{\mathrm{NS}}$ \\
\hline & 1 & & & & & & & & & & & 25 & $0.428^{*}$ \\
\hline TP & 2 & & & & & & & & & & & 27 & $0.136^{\mathrm{NS}}$ \\
\hline TP & 3 & & & & & & & & & & 1.000 & 22 & $-0.303^{\mathrm{NS}}$ \\
\hline & $\mathrm{T}$ & & & & & & & & & & & 74 & $-0.001^{\mathrm{NS}}$ \\
\hline
\end{tabular}

H: Herd, T: Total, UIBC $=$ unsaturated iron binding capacity, $\mathrm{TIBC}=$ total iron-binding capacity, TSAT $=$ transferrin saturation, $\mathrm{NS} \mathrm{P}>0.05 * \mathrm{P}<0.05$ $* * \mathrm{P}<0.01 * * * \mathrm{P}<0.001$ 
Chloride: In this study, the mean $\mathrm{Cl}$ value of the total herd was $111.105 \pm 0.582 \mathrm{mmol} / \mathrm{L}$. Moreover, the minimum-maximum $\mathrm{Cl}$ values ranged from 96.000 to $140.600 \mathrm{mmol} / \mathrm{L}$, and a fairly high level statistical significant $(\mathrm{P}<0.001)$ was between 3 Honamli herds.

In the study conducted by Piccione et al. (2010), determining the reference values in Girgentana goats, $\mathrm{Cl}$ levels varied $103.40 \pm 1.09 \mathrm{mmol} / \mathrm{L}, 103.90 \pm 0.56 \mathrm{mmol} / \mathrm{L}$ and $106.60 \pm 1.45 \mathrm{mmol} / \mathrm{L}$ in 3 groups $(1-2,3-4$ and $5-6$ years), respectively. This indicates that the age factor did not significantly affect the $\mathrm{Cl}$ level, but the $\mathrm{Cl}$ level had a fairly high level statistical significant $(\mathrm{P}<0.001)$ between 3 Honamli herds in this study.

Potassium: In this presented study, the mean $\mathrm{K}$ value of the total herd was $4.519 \pm 0.066 \mathrm{mmol} / \mathrm{L}$. Moreover, the minimum-maximum $\mathrm{K}$ values ranged from 3.250 to 6.350 $\mathrm{mmol} / \mathrm{L}$, and a statistical significant $(\mathrm{P}<0.05)$ was between 3 Honamli herds.

The control group of Altug et al. (2013) had a higher value $\mathrm{Na}(5.30 \pm 0.55 \mathrm{mEq} / \mathrm{L})$ than the presented study's value $(4.519 \pm 0.066 \mathrm{mmol} / \mathrm{L})$.

Magnesium: In this study, the mean $\mathrm{Mg}$ value of the total herd was $2.161 \pm 0.057 \mathrm{mg} / \mathrm{dL}$. Moreover, the minimum-maximum $\mathrm{Mg}$ values ranged from 3.250 to $6.350 \mathrm{mg} / \mathrm{dL}$, and a high statistical significant $(\mathrm{P}<0.01)$ was between 3 Honamli herds. Devrim et al. (2015) detected that 12 months old Honamli goats had both $3.03 \pm 0.12 \mathrm{mg} / \mathrm{dL} \mathrm{Mg}$ level and a statistical significant $(\mathrm{P}<0.05)$ between 3 groups. Besides, Sovende et al. (2008) reported that the blood minerals of Wad goats grazing in the natural habitat were $\mathrm{Mg} 0.72 \pm 0.03 \mathrm{mmol} / \mathrm{L}$. On the other hand, Fujihara et al. (2006) reported that $\mathrm{Mg}$ levels were $30.05 \pm 1.11 \mathrm{mg} / \mathrm{L}$ and $29.40 \pm 1.09 \mathrm{mg} / \mathrm{L}$, in the dry and wet season respectively. Fujihara et al. (2006) reported higher $\mathrm{Mg}$ values than reported by McDowell (1985) as $\mathrm{Mg} 15 \mathrm{mg} / \mathrm{L} . \mathrm{Mg}$ levels in the total of 3 herds were found to be lower compared to the ones reported by Fujihara et al. (2006), while results were higher than critical levels reported by McDowell (1985) in the presented study. In spite of the fact that, there is no difference between the results Devrim et al. (2015), Fujihara et al. (2006) and this study.

Sodium: In this presented study, the mean Na value of the total herd was $148.047 \pm 0.508 \mathrm{mmol} / \mathrm{L}$. Moreover, the minimum-maximum $\mathrm{Na}$ values ranged from 132.000 to $176.000 \mathrm{mmol} / \mathrm{L}$, and no statistical significant $(\mathrm{P}>0.05)$ was between 3 Honamli herds.

In a study conducted by Altug et al. (2013) on Native goats with chronic fluorosis, the serum $\mathrm{Na}$ level of the control group was determined as $142.1 \pm 11.5 \mathrm{mEq} / \mathrm{L}$. These two study's results overlap with each other.

Iron: In this study, the mean Fe value of the total herd was $148.047 \pm 0.508 \mu \mathrm{g} / \mathrm{dL}$. Moreover, the minimummaximum $\mathrm{Fe}$ values ranged from 132.000 to 176.000 $\mu \mathrm{g} / \mathrm{dL}$, and a statistical significant $(\mathrm{P}<0.05)$ was between 3 Honamli herds.

AL-Dujaily and AL-Hadithy (2014) explained Fe level of normal goats as $11.8 \pm 0.22 \mu \mathrm{mol} / \mathrm{L}$. When this unit is translated, the following result is obtained: $244.306 \mu \mathrm{g} / \mathrm{dL}$. Which can be said to be quite high compared to the value obtained as a result of this study. On the other hand, Devrim et al. (2015) reported that no statistical difference (P>0.05) between the level of blood Fe levels was in the 4,
8 and 12 month old Honamli goats. At the same time, 12 month old goats blood Fe levels was defined as $143.3 \pm 3.91$ $\mu \mathrm{g} / \mathrm{dL}$ by them. Which coincides with the result of the study and can be used to refer to the value of healthy goats.

$U I B C$ : In this presented study, the mean UIBC value of the total herd was $155.025 \pm 4.333 \mu \mathrm{g} / \mathrm{dL}$. Moreover, the minimum-maximum UIBC values ranged from 61.300 to $250.500 \mu \mathrm{g} / \mathrm{dL}$, and a high statistical significant $(\mathrm{P}<0.01)$ was between 3 Honamli herds.

There is no evidence in the literature about the value of UIBC in goats. In a study conducted in sheep, serum Fe level has been associated with birth. And in the same study, Cihan et al. (2016) calculated UIBC levels as 265.7 \pm 26.3 $\mu \mathrm{g} / \mathrm{dL}$ in non-pregnant sheep. In another study conducted on Awassi sheep, this level was $149 \pm 5 \mu \mathrm{g} / \mathrm{dL}$ (Al-Hadty and Al-Badawi, 2012). Based on this, it can be said that the result of this study is closer to the result of Awassi sheep.

TIBC: In this study, the mean TIBC value of the total herd was $267.051 \pm 4.570 \mu \mathrm{g} / \mathrm{dL}$. Moreover, the minimummaximum TIBC values ranged from 166.400 to 361.400 $\mu \mathrm{g} / \mathrm{dL}$, and a fairly high statistical significant $(\mathrm{P}<0.001)$ was between 3 Honamli herds.

In the study conducted by AL-Dujaily and AL-Hadithy (2014) for the evaluation of some biochemical parameters in anemic goats, the TIBC level in the control group of female goats was $90.1 \pm 0.86 \mu \mathrm{mol} / \mathrm{L}$. It was found that ALDujaily and AL-Hadithy (2014) had 7 times more TIBC value on the control group's goats than this study result when the units were converted $(1 \mu \mathrm{g} / \mathrm{dL}=20.703934$ $\mu \mathrm{mol} / \mathrm{L})$ of these two results. Against this, Cihan et al. (2016), found that TIBC values of non-pregnant sheep were $477.8 \pm 13.6 \mu \mathrm{g} / \mathrm{dL}$, which supports that the TIBC result of this study can be referenced.

TSAT: In this presented study, the mean TSAT value of the total herd was $41.989 \pm 1.034 \%$. Moreover, the minimum-maximum TSAT values ranged from 22.930 to 74.060 , and no statistical significant $(\mathrm{P}>0.05)$ was between 3 Honamli herds.

Also, AL-Dujaily and AL-Hadithy (2014) calculated the TSAT was $13.1 \pm 0.25 \%$ in normal goats, and they have found that this level has fallen considerably in irondeficient anaemic goats $(4.1 \pm 0.23 \%)$. Also, the value already presented in this study was close to this reference value of normal goats.

$T f$ : In this study, the mean Tf value of the total herd was $171.274 \pm 3.666 \mathrm{~g} / \mathrm{L}$. Moreover, the minimum-maximum Tf values ranged from 90.120 to $246.120 \mathrm{~g} / \mathrm{L}$, and no statistical significant $(\mathrm{P}>0.05)$ was between 3 Honamli herds.

There is no evidence in the literature about the value of Tf in goat. But, Tf measurement at the body is very important for detection of serum Fe concentration, iron saturation and particularly body iron deposits. Despite this, serum $\mathrm{Fe}$ and TIBC values not included in the sensitive tests are widely used to determine the Fe status. In addition, serum $\mathrm{Fe}$ is known to be a direct measure of transferrinbound iron. For this reason, TIBC is considered as an indirect measure of $\mathrm{Tf}$ concentration. While a small number of studies have been found related with $\mathrm{Tf}$ level (Thoren-Tholling and Martinsson, 1974; Lampreave and Pineiro, 1992; Ilic et al., 2006), many other researchers (Kozat et al., 2006; Eltain 2008; Merhan and Ozcan, 2010; AL-Dujaily and AL-Hadithy, 2014) prefer to use the TSAT value in their study. 
$T P$ : In this presented study, the mean TP value of the total herd was $7.055 \pm 0.120 \mathrm{~g} / \mathrm{dL}$. Moreover, the minimummaximum TP values ranged from 2.730 to $9.410 \mathrm{~g} / \mathrm{dL}$, and no statistical significant $(\mathrm{P}>0.05)$ was between 3 Honamli herds.

Cepeda-Palacios et al. (2018) reported that the blood serum TP level was $8.1 \pm 0.8 \mathrm{~g} / \mathrm{dL}$ in total herd, when the post-kidding group TP values was $7.4 \pm 1 \mathrm{~g} / \mathrm{dL}$. Also this study result was quite close to result declared by CepedaPalacios et al. (2018). These researchers declared that there was no statistical significant $(\mathrm{P}>0.05)$ between breed groups (Anglonubian, Saanen X Anglonubian), whereas physiological stage (pregnancy, post-kidding, control) had a statistical significant $(\mathrm{P}<0.05)$. Here, pregnancy and control groups had similar values and TP value in the postkidding group decreased was saw. Chen et al. (1999) reported that the reduction of blood serum protein (TP), especially $\gamma$-globulin, in Nubian goats promotes breast secretion. Although the goat breeds studied are different, Tp values of this study were similar declared by CepedaPalacios et al. (2018). Kaneko et al. (1997) reported that change in serum proteins levels with age is an important in the interpretation of serum proteins. In corroborates this opinion, no statistical significant $(\mathrm{P}>0.05)$ was between 3 Honamli herds in the same age group (adult) related with TP levels of this study.

$\mathrm{Ca}++$ : In this study, the mean $\mathrm{Ca}++$ value of the total herd was $2.953 \pm 0.086 \mathrm{mg} / \mathrm{dL}$. Moreover, the minimummaximum $\mathrm{Ca}++$ values ranged from 0.924 to $4.437 \mathrm{mg} / \mathrm{dL}$, and no statistical significant $(\mathrm{P}>0.05)$ was between 3 Honamli herds. Also, The importance of determining $\mathrm{Ca}++$ level is that $\mathrm{Ca}$ is the physiologically active form and 50$55 \%$ of total $\mathrm{Ca}$ is found in this form.

Studies on the biochemical and haematological values of goats still continue, but still less than the number of sheep. However, these values of animals are very important in terms of nutrition, reproductive characteristics and relation to disease. Moreover, as presented in this study, besides mineral values, formula calculations, ratio of minerals to each other, mineral ranking and finally correlation values of the data can be needed in interpreting the literature. As a result, serum mineral levels at Honamli goats were within the physiological reference ranges in this presented study. Assuming no difference between the herds, it is considered that the results of the analyses are important in terms of expressing the clinical values of healthy Honamli goats and will be a reference to new studies.

\section{References}

AL-Dujaily AH, AL-Hadithy H AH. 2014. Determination of some biochemical parameters in clinically healthy and anemic goats. Veterinary Medical Sciences, 5(2):168-178, DOI: 10.13140/RG.2.2.20938.41928

Aktas AH, Gok B, Ates S, Tekin ME, Halici I, Bas H, Erduran H, Kassam S. 2015. Fattening performance and carcass characteristics of Turkish indigenous Hair and Honamli goat male kids. Turkish Journal of Veterinary and Animal Sciences. 39(6):643-653, DOI: 10.16971/vd.78142

AL-Hadty HAH, AL-Badawi NM. 2012. Evaluation of specific biochemical values in clinically normal and anemic Awassi sheep. International Journal of Science and Nature. 3:688-691.
Alizadehasl M, Unal N. 2011. Kilis, Norduz ve Honamli keçilerinde bazı morfolojik özellikler. Journal of Lalahan Livestock Research Institute. 51(2): 81-92.

Altuğ N, Arslan S, Yüksek N, Keleş I, Yörük IH, Başbuğan Y, Aytekin I. 2013. The levels of trace elements and selected vitamins in goats with chronic fluorosis. Turkish Journal of Veterinary and Animal Sciences. 37(5):529-534. DOI: 10.3906/vet-1302-2

Anonymous, 2018a. Available from: http://www2.luresext.edu/ goats/training/nutrition.pdf. [Accessed 16 February 2010].

Anonymous, 2018b. Available from: http://www.archem.com.tr/ Upload/Dosyalar/8765069842u.pdf. [Accessed 16 February 2010].

Anonymous, 2018c. Available from: http://www.multiquimicos.com/ plasmatecpdfs/TOTAL_IRON-BINDING_CAPACITY.pdf. [Accessed 16 February 2010].

Bieglmayer C, Chan DW, Sokoll L, Imdahl R, Kobayashi M, Yamada E, Lilje DJ, Luthe H, Meissner J, Messeri G, Celli A, Tozzi P, Roth HJ, Schmidt FP, Mächler ML, SchuffWerner P, Zingler C, Smitz J, Schiettecatte J, Vonderschmitt DJ, Pei P, Ng K, Ebert C, Kirch P, Wanger M, McGovern M, Stockmann W, Kuns A. 2004. Multicentre Evaluation of the E170 Module for MODULAR ANALYTICS. Clin. Chem. Lab. Med., 42, 1186-1202

Cepeda-Palacios R, Fuente-Gomez MG, Ramirez-Orduna JM, Garcia-Alvarez A, Lnias- Cervantes X, Angulo C. 2018. Effects of pregnancy and post-kidding stages on haematochemical parameters in cross-bred goats. Journal of Applied Animal Research. 46(1):269-273, DOI: 10.1080/09712119.2017.1295970

Chen JC, Chang CJ, Peh HC, Chen SY. 1999. Blood serum protein levels and neonatal growth rate of Nubian goat kids in Taiwan area. Small Ruminant Research. 32:153-160.

Cihan H, Temizel EM, Yilmaz Z, Ozarda Y. 2016. Serum iron status and its relation with haematological indexes before and after parturition in sheep. Journal of the Faculty of Veterinary Medicine, Kafkas University. 22(5),679-683. DOI: 10.9775/kvfd.2016.15103

Demir AO, Mert N, Karakus F, Bingol M, Kor A. 2011. Farkli isletme kosullarinda yetistirilen Ankara kecilerinde tiftik kalitesi ve kan serumundaki bazi mineral madde duzeylerinin arastirilmasi. 7. Ulusal Zootekni Bilim Kongresi, Adana, Turkiye.

Devrim AK, Elmaz O, Mamak N, Sudagidan M. 2015. Alterations in some clinical biochemistry values of Honamli and Native Hair goats during pubertal development. Veterinarski Arhiv. 85(6):647-656, ISSN 0372-5480

Dlouhy AC, Outten CE. 2013. Banci, Lucia, ed. Metallomics and the cell. metal ions in life sciences. 12. Springer. DOI:10.1007/978-94-007-5561-18. ISBN 978-94-007-5560-4.

Elmaz O, Saatc1 M, Mamak N, Dag B, Aktas AH, Gok B, 2012a. The determination of some morphological characteristics of Honamli goat and kids, defined as a new indigenous goat breed of Turkey. Kafkas Universitesi Veteriner Fakultesi Dergisi, 18, 481-485. DOI: 10.9775/kvfd.2011.5767

Elmaz O, Saatci M, Dag B, Aktas AH, Ata A, Gulay MS, Mamak N, Gok B. 2012b. Some descriptive characteristics of a new goat breed called Honamli in Turkey. Tropical Animal Health and Production. 44(8):1913-1920, DOI: 10.1007/s11250012-0156-7.

Eltain MIA. 2008. Changes in haematology iron metabolism and body weight in sheep fed by nigella sativa oil supplement. Master Thesis. Department of Biochemistry, Faculty of Veterinary Medicine, University of Khartoum.

Fujihara T, Serra AB, Serra SD, Orden EA. 2006. Mineral nutrition of grazing goats in Luzon Island, Philippines. Bulletin of the Faculty of Life and Environmental Science. Shimane Univ., 1:19-34. 
Gok B, Aktas AH, Bas H, Dursun S, Halici I, 2014. Islah calısması yurutulen Honamli kecisi surulerindeki keclerin dol verimi ve canli agirliklari ile oglaklarin buyume ve yasama gucu ozellikleri. Uluslararasi Mezopotamya Tarim Kongresi, Diyarbakir, Turkiye, pp: 264- 265.

Gok B, Aktas AH, Dursun S, 2011. Rising star of the Taurus Mountains: Honamli goats. RBI 8th Global Conference on the Conservation of Animal Genetic Resources, Tekirdag, Turkey, pp: 65-72.

Gok B, Dursun S, Aktas AH, Halici I, Bas H, 2014b. Growth performance and body measurement of Honamli goat kids protected as a native anImal genetic resource kept in breeder conditions in Turkey. European Regional Conference on Goats, Debrecen, Macaristan, p: 58.

Gok B, Aktas AH, Halici I, Bas H. 2015. Halk elinde koruma altina alinan Honamli kecisi ve oglaklarinin canli agirliklari ve bazi vucut olculeri. Eurasian Journal of Veterinary Sciences. 31: 4, 227-234. DOI: 10.15312/EurasianJVetSci. 2015413528

Ilic V, Petakov M, Stojanovic N, Jovcic G, Bugarski D, Grbovic T, Bozic T, Kovacevic-Filipovic M. 2006. Relationship between total iron binding capacity and transferrin concentration in neonatal piglets treated with iron dextran. Acta Veterinaria (Beograd), 56(2-3)235-242. DOI: 10.2298/AVB0603235I

Kaneko JJ, Harvey JW, Bruss ML, 1997. Clinical biochemistry of domestic animals. Academic Press, Inc., San Diego, London, Boston, New York, Sydney. ISBN: 9780080529196

Kawas JR, Andrade-Montemayor H, Lu CD. 2010. Strategic nutrient supplementation of free-ranging goats. Small Ruminant Research. 89(2): 234-243. DOI: 10.1016/ j.smallrumres.2009.12.050

Kozat S, Yuksek N, Goz Y, Keles I. 2006. Serum iron, total ironbinding capacity, unbound iron-binding capacity, transferrin saturation, serum copper and hematological parameters in pregnant Akkaraman ewes infected with gastro-intestinal parasites. Turkish Journal of Veterinary and Animal Sciences. 30(6):601-604. ISSN:1300-0128

Kunish JP., Small LL 1970. Clinical Chemistry. 16, 148-150.

Lampreave F, Pineiro A. 1992 Concentration of major plasma proteins in serum and whole tissue extracts of porcine fetuses during development. Journal of Reproduction and Fertility. 95(2):441-9. DOI: 10.1530/jrf.0.0950441.
McDowell LR.1985. Nutrition of grazing ruminants in warm climates. pp.168-169.

AcademicPress Inc. San Diego, C.A., USA. eBook ISBN: 9780323138871

Merhan O, Ozcan A. 2010. Investigation of ceruloplasmin, haptoglobin, fibrinogen, albumin and transferrin levels of sheep in the peripartum period. Journal of Faculty of Veterinary Medicine, Erciyes University 7(1):13-20. ISSN 1304-7280

Ogunleke FO, Ajayi FT, Morakinyo OA, Saka AA. 2014. Performance and blood profile of West African Dwarf Goat feed concentrate supplement containing varying levels of corn cobs. American Journal of Experimental Agriculture. 4(12):1861-1868. DOI: 10.9734/AJEA/2014/11271

Patkowska-Sokola B, Dobrzanski Z, Osman K, Bodkowski R, Zygadlik K. 2009. The content of chosen chemical elements in wool of sheep of different origins and breeds. Archiv Tierzucht 52(4):410-418. DOI: 10.5194/aab-52-410-2009

Piccione G, Casella S, Lutri L., Vazzana I, Ferrantelli V, Caola G. 2010. Reference values for some haematological, haematochemical and electrophoretic parameters in the Girgentana goat. Turkish Journal of Veterinary and Animal Sciences. 34(2): 197-204. DOI:10.3906/vet-0902-1

Raphael SS. 1983. Lynch's Medical Laboratory, Technology.4th ed. W.B.Saunders. p 261. ISBN-13: 978-0721674650

Sowande OS, Odufowora EB, Adelakun AO, Egbeyale LT. 2008. Blood minerals in Wad sheep and goats grazing natural pastures during wet and dry seasons. Archivos de Zootecnia. 57(218):275-278. ISSN: 0004-0592

Thoren-Tholling K, Martinsson K. 1974. On the transferrin concentration in serum of sows and growing pigs and in sow colostrum. Acta Veterinaria Scandinavica, 15, 120-34.

Xin GS, Longa RJ, Guo XS, Irvine J, Ding IM, Ding II, Shanga ZH. 2011. Blood mineral status of grazing Tibetian sheep in the Northeast of the Qighai-Tibetian Plateu. Livestock Science. 136, 102-107. DOI: 10.1016/j.livsci.2010.08.007

Yee GM, Tolman WB. 2015. Sustaining life on planet earth: metalloenzymes mastering dioxygen and other chewy gases. metal ions in life sciences. Kroneck Peter MH, Sosa Torres ME, eds. 15. Springer. pp.131-204. Doi:10.1007/978-3-31912415-5_5. ISBN 978-3-319-12415-5 